\title{
Coherent terahertz radiation emitted by wide-angle electron beams from a laser-wakefield accelerator
}

\author{
Enrico Brunetti ${ }^{\mathrm{a}}$, Xue Yang ${ }^{\mathrm{b}}$, and Dino A. Jaroszynski ${ }^{\mathrm{a}}$ \\ aSUPA, Department of Physics, University of Strathclyde, Glasgow, G4 0NG, UK \\ ${ }^{b}$ Department of Physics, Capital Normal University, Key Lab of Terahertz Optoelectronics, \\ Ministry of Education, and Beijing Advanced Innovation Center for Imaging Technology, \\ Beijing 100048, China
}

\begin{abstract}
Laser-wakefield accelerators generate femtosecond-duration electron bunches with energies from $10 \mathrm{~s}$ of $\mathrm{MeV}$ to several $\mathrm{GeV}$ in millimetre distances by exploiting the large accelerating gradients created when a high-intensity laser pulse propagates in an underdense plasma. The process governing the formation of the accelerating structure ("bubble") also causes the generation of sub-picosecond duration, 1-2 MeV nanocoulomb electron beams emitted obliquely into a hollow cone around the laser propagation axis. We present simulations showing that these wide-angle beams can be used to produce coherent transition radiation in the 0.1-5 $\mathrm{THz}$ frequency range with $10 \mathrm{~s} \mu \mathrm{J}$ to mJ-level energy if passed through an inserted metal foil, or directly at the plasma-vacuum interface. We investigate how the properties of terahertz radiation change with foil size, position and orientation. The bunch length and size of wide-angle beams increase quickly as the electrons leave the accelerator, causing a shift of the radiation frequency peak from about $1 \mathrm{THz}$ at a distance of $0.1 \mathrm{~mm}$ from the accelerator exit to $0.2 \mathrm{THz}$ at $1 \mathrm{~mm}$. If the foil size is reduced, for example to match the typical diameter of the plasma channel formed in a laser-wakefield accelerator, simulating the emission from the plasma-vacuum boundary, the low-frequency side of the spectrum is suppressed. The charge of wide-angle electron beams is expected to increase linearly with the laser intensity, with a corresponding quadratic increase of the terahertz radiation energy, potentially paving the way for mJ-level sources of coherent terahertz radiation.
\end{abstract}

Keywords: Terahertz generation, laser-wakefield acceleration

\section{INTRODUCTION}

Terahertz $(\mathrm{THz})$ radiation, which lies between 0.1 and $10 \mathrm{THz}(30-3000 \mu \mathrm{m})$ in the electromagnetic spectrum, is of interest for many applications, such as chemical and biological imaging, communication and quality control. ${ }^{1-4}$ There are many sources of $\mathrm{THz}$ radiation, but few of them produce high-intensity pulses suitable for driving non-linear optical effects in materials. ${ }^{5}$ One source of intense THz pulses is coherent transition radiation (CTR) emitted by an electron beam passing through a thin metallic foil. ${ }^{6}$ If the bunch length is shorter than the radiation wavelength, the contribution from all the electrons add coherently and the total radiated power scales as the square of the beam charge. Assuming a longitudinally and transversally Gaussian bunch, the spectrum of CTR can be calculated analytically in the far-field approximation. ${ }^{7}$ As shown in Figure 1, efficient generation of CTR with frequency up to $10 \mathrm{THz}$ is possible for bunch durations shorter than about $100 \mathrm{fs}$, which are achievable using conventional accelerators equipped with bunch compressors or other beam manipulation devices. ${ }^{8}$ Laserdriven electron accelerators, ${ }^{9}$ on the other hand, directly produce bunches with duration as short as $1-5$ fs. ${ }^{10,11}$ Recent experiments have shown, for example, that electron beams with energy of $250 \mathrm{MeV}$, charge of $250 \mathrm{pC}$, divergence of $7 \mathrm{mrad}$ and an estimated bunch length of 5 fs can be produced reliably using self-truncated ionisation injection. ${ }^{12}$ If focused down onto a metallic foil to a radius of $50 \mu \mathrm{m}$, this beam would produce broadband CTR with $1 \mathrm{~mJ}$ energy in the $0.1-20 \mathrm{THz}$ spectral range.

Laser-wakefield accelerators have been shown not only to produce high-energy, short electron bunches, but also to emit a low-energy, high-charge electron beam on a cone centred along the laser propagation axis. ${ }^{13,14}$

Correspondence can be sent to DAJ. E-mail: d.a.jaroszynski@strath.ac.uk 


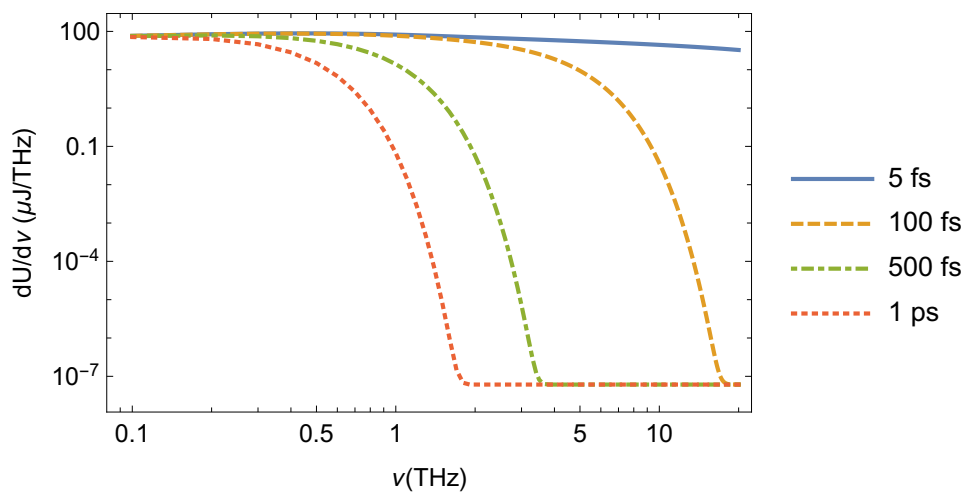

Figure 1. Spectrum of CTR generated by a $250 \mathrm{MeV}$ Gaussian electron bunch with charge of $250 \mathrm{pC}$, transverse radius $(\sigma)$ of $50 \mathrm{\mu m}$ and duration (FWHM) of $5 \mathrm{fs}, 100 \mathrm{fs}, 500 \mathrm{fs}$ and $1 \mathrm{ps}$.

Theoretical studies indicate that these wide-angle beams can also produce mJ-level THz radiation. ${ }^{15}$ Here we present numerical simulations to investigate the properties of this $\mathrm{THz}$ source. We show that the bunch duration of wide-angle beams is predicted to be longer than what is obtained from measurements and simulations of highenergy forward electron beams, resulting in the suppression of CTR at frequencies above 1-3 THz, depending on the geometry. The large beam charge, however, still enables to reach mJ-level energies in the $0.1-3 \mathrm{THz}$ spectral range.

\section{RESULTS}

A schematic diagram of a possible setup to produce $\mathrm{THz}$ radiation using wide-angle electron beams is presented in Figure 2. A laser beam is focused onto a gas or plasma target to an intensity in excess of $1 \times 10^{18} \mathrm{~W} / \mathrm{cm}^{2}$, generating a high-energy forward electron beam along the laser propagation axis and a low-energy electron beam in a cone around the laser axis. A foil intercepts the wide-angle beam at an offset $d$ and angle $\alpha$ from the laser axis. Transition radiation is emitted in the backward and forward direction and observed on a detector at a distance $R$ from the centre of the foil. Here we have chosen $R=500 \mathrm{~mm}$, which corresponds to a realistic value for an experiment. The laser-plasma interaction has been modelled with the particle-in-cell (PIC) code OSIRIS. ${ }^{16}$ The properties of wide-angle electron beams have been found to vary little with laser and plasma parameters for a large range of values. ${ }^{13}$ Here, as a representative example, we present the results of a three-dimensional simulation of a horizontally polarised laser with $0.8 \mu \mathrm{m}$ wavelength, normalised vector potential $a_{0}=3$, spot radius $w_{0}=7 \mu \mathrm{m}$ and $20 \mathrm{fs}$ pulse duration interacting with a pre-ionised plasma with density $2 \times 10^{19} \mathrm{~cm}^{-3}$ and $500 \mu \mathrm{m}$ length. These parameters reproduce experiments presented in Ref. 13. A sample of 20,000 electrons has been selected from the phase-space distribution produced by OSIRIS and used to calculate numerically transition radiation from the foil using a vector model described in Ref. 15 and 17.

Figure 3 shows the spatial profile and the energy spectrum of wide-angle electrons as they hit a $1 \mathrm{~mm} \times 1 \mathrm{~mm}$ foil at an offset $d=100 \mu \mathrm{m}$ from the laser axis and orientation $\alpha=60^{\circ}$. The mean electron energy is $1.8 \mathrm{MeV}$ with an exponential distribution. The beam size (FWHM) is $144 \mu \mathrm{m}$ in the horizontal direction $(x)$ and $65 \mu \mathrm{m}$ in the vertical direction $(y)$. The bunch duration (FWHM) in the direction perpendicular to the foil surface is $160 \mathrm{fs}$. As the beam propagates further away from the accelerator, the transverse and longitudinal size grow quickly, as shown in Figure 4, which corresponds to the electron distribution on a $3 \mathrm{~mm} \times 3 \mathrm{~mm}$ foil placed at offsets $d=100 \mu \mathrm{m}, d=500 \mu \mathrm{m}$ and $d=1 \mathrm{~mm}$, and orientations $\alpha=60^{\circ}$ and $\alpha=0$ (parallel to the laser axis). For $d=1 \mathrm{~mm}$ and $\alpha=60^{\circ}$ the beam size is $630 \mu \mathrm{m}$ in the horizontal direction and $310 \mu \mathrm{m}$ in the vertical direction with a bunch duration of $410 \mathrm{fs}$. If the foil is parallel to the laser axis the beam size is $910 \mu \mathrm{m}$ in the horizontal direction and $555 \mu \mathrm{m}$ in the vertical direction with a bunch duration of $2.3 \mathrm{ps}$.

The modelling of CTR emitted by wide-angle electrons passing through the foil requires a numerical approach to account for the complex electron bunch shape. Here we use a vector model that is valid for arbitrary incidence angles and directly uses the phase-space electron distribution obtained from PIC simulations. Figure 5 shows the spectrum of CTR generated on a $3 \mathrm{~mm} \times 3 \mathrm{~mm}$ foil with orientation $\alpha=0$ and $\alpha=60^{\circ}$ for different offsets $d$ from 


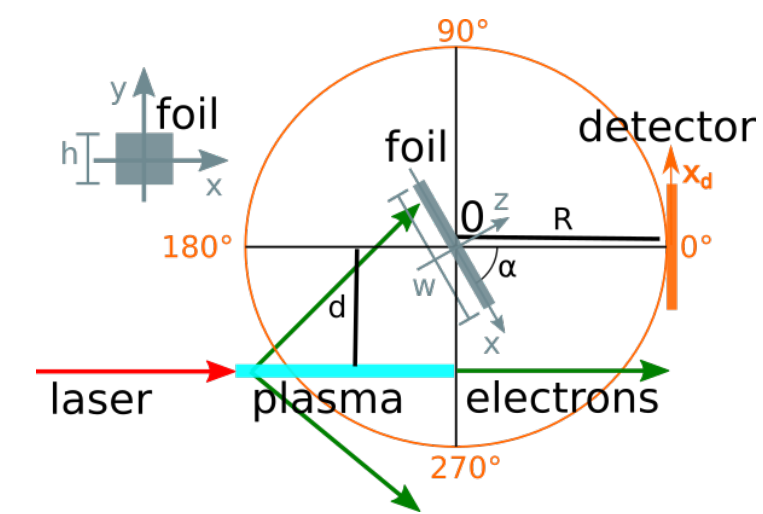

Figure 2. Schematic diagram of a $\mathrm{THz}$ source based on laser-driven wide-angle electron beams passed through a thin metallic foil.
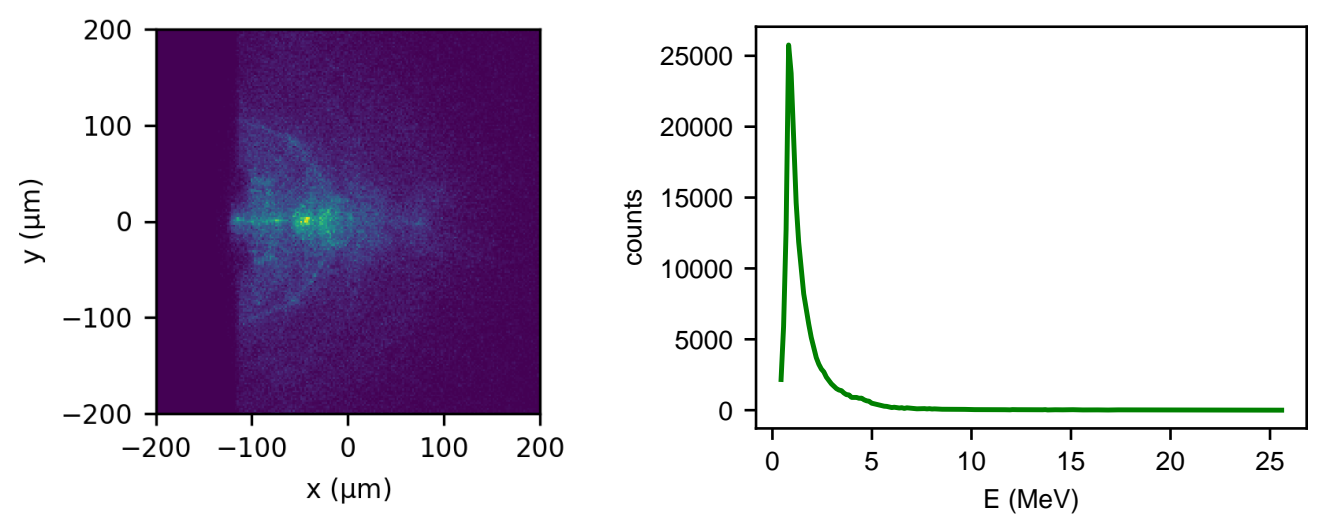

Figure 3. Spatial profile (left) and spectrum (right) of wide-angle electrons on a $1 \mathrm{~mm} \times 1 \mathrm{~mm}$ foil with $60^{\circ}$ orientation and $100 \mu \mathrm{m}$ offset from the laser axis. Plots represent a sample of 200,000 particles obtained from OSIRIS simulations.

the laser axis, assuming an electron beam charge of $1 \mathrm{nC}$ and observation angle of $0^{\circ}$. The integrated radiation energy for $d=100 \mu \mathrm{m}$ in a $45^{\circ}$ cone is $295 \mu \mathrm{J}$ for $\alpha=0$ and $56 \mu \mathrm{J}$ for $\alpha=60^{\circ}$. Simulations indicate that the charge of wide-angle electron beams scales linearly with laser intensity and can exceed $10 \mathrm{nC}$ using commercially available lasers. ${ }^{13}$ The CTR energy scales with good approximation as the square of the charge, ${ }^{13}$ allowing to reach mJ-level energies.

\section{DISCUSSION}

Laser-wakefield accelerators produce 100s MeV electron bunches with femtosecond duration, mrad divergence and up to 100s pC charge that can drive mJ-level THz sources based on CTR from a thin metallic foil. Intense THz radiation can also be produced using the low-energy, high-charge electrons that laser-wakefield accelerators emit over a wide-angle cone around the laser propagation axis. The divergence of transition radiation is approximately $1 / \gamma$, with $\gamma$ the relativistic factor of the electrons. For low energy, as in wide-angle beams, radiation is emitted over a large angle, of the order of $40^{\circ}$ (FWHM), which may require re-focusing and may not be desirable for some applications. An advantage of low electron energies, on the other hand, is that the formation length of forward transition radiation is about $1 \mathrm{~mm}$ at $1 \mathrm{THz}$, in contrast to a formation length longer than $10 \mathrm{~m}$ for a $250 \mathrm{MeV}$ electron beam. ${ }^{18}$ For both forward and oblique electron beams, the THz source can be realised in practice by inserting a thin metallic foil very close to the accelerator exit. However, in this region the laser intensity is still very high and the foil is likely to be quickly damaged. High-energy electron beams could be transported and focused further downstream at a distance where laser damage is no longer an issue using high-gradient permanent quadrupoles ${ }^{19}$ or plasma lenses. ${ }^{20,21}$ Wide-angle electrons, on the other hand, are not co-propagating with the 

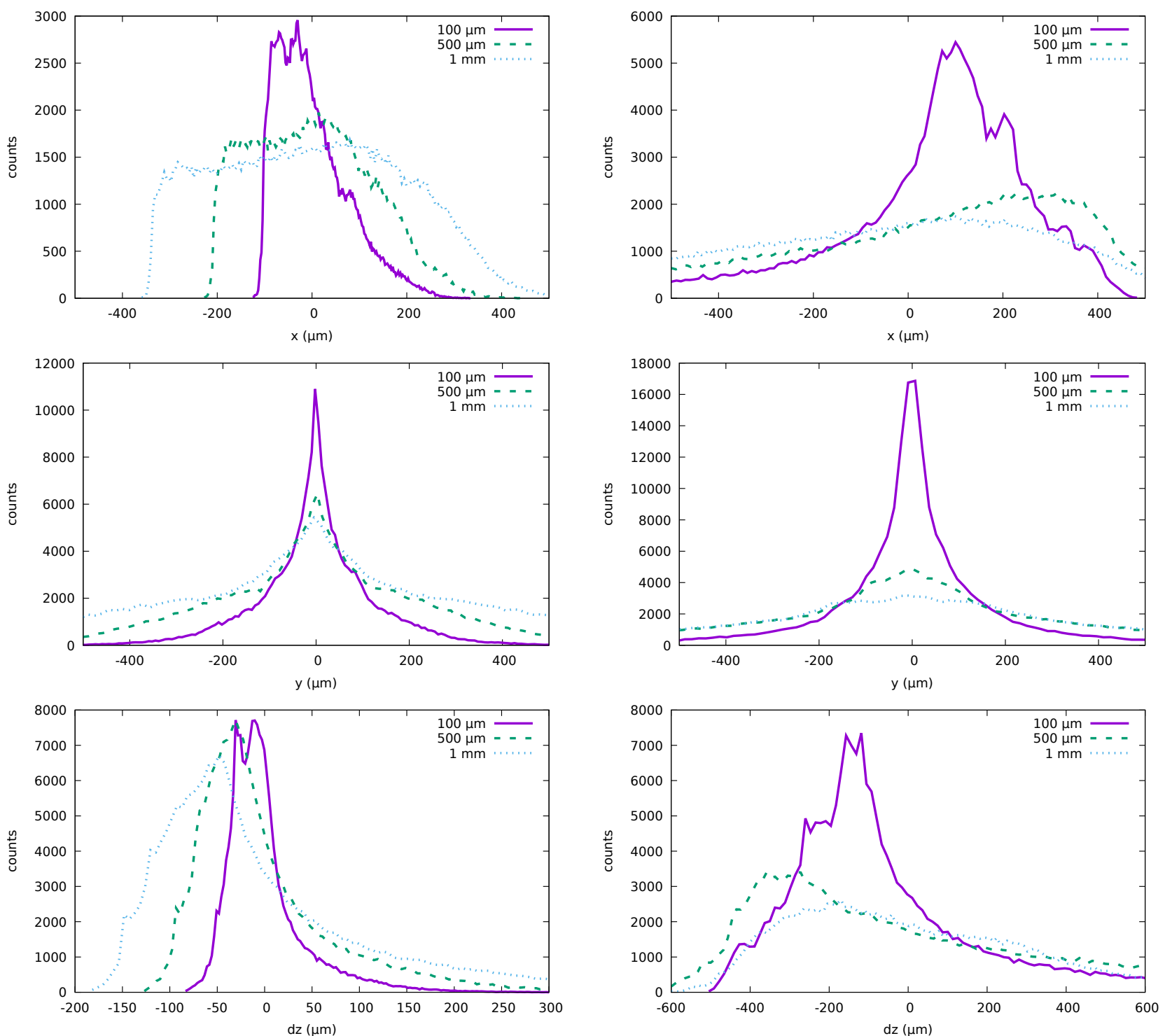

Figure 4. Horizontal (top), vertical (middle) and longitudinal (bottom) profile of a wide angle electron beam intercepting a $3 \mathrm{~mm} \times 3 \mathrm{~mm}$ foil with $60^{\circ}$ (left) and parallel (right) orientation with respect to the laser propagation axis at an offset of $100 \mu \mathrm{m}, 500 \mu \mathrm{m}$ and $1 \mathrm{~mm}$. Plots represent a sample of 200,000 particles obtained from OSIRIS simulations. 

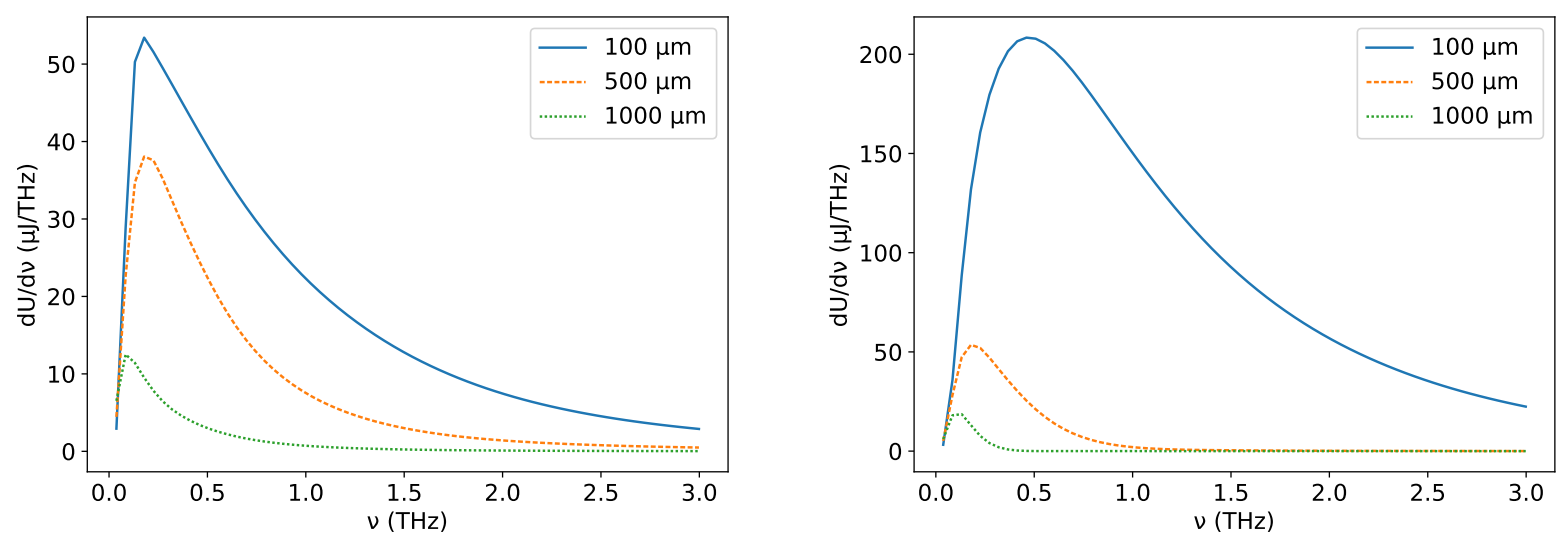

Figure 5. Spectrum of CTR emitted by a $1 \mathrm{nC}$ wide-angle electron beam passing through a $3 \mathrm{~mm} \times 3 \mathrm{~mm}$ foil with $60^{\circ}$ (left) and parallel (right) orientation for varying offset. Plots are calculated from a sample of 20,000 particles obtained from OSIRIS simulations.

laser beam, and could be passed through a foil placed off-axis, avoiding the intense part of the laser beam. The quick beam expansion, however, will lead to the suppression of the high frequency part of the emitted CTR. Wide-angle electron beams can also produce radiation directly at the plasma-vacuum boundary, which can act as a metallic interface. ${ }^{22}$ In this case a suppression of the low frequency part of the spectrum is expected because the transverse size of the plasma $(100 \mathrm{~s} \mu \mathrm{m})$ can be smaller than the radiation wavelength.

In conclusion, we have shown that wide-angle electrons emitted by laser-wakefield accelerators can produce $\mathrm{THz}$ radiation with $10 \mathrm{~s} \mu \mathrm{J}$ to $\mathrm{mJ}$-level energy in the $0.1-5 \mathrm{THz}$ spectral range, depending on the laser parameters and experimental geometry. Radiation can be produced by inserting a metallic foil close to the accelerator exit or directly at the plasma-vacuum boundary. In the latter case THz radiation could be an unwanted background noise in experiments, requiring proper shielding.

\section{ACKNOWLEDGMENTS}

The authors thank the OSIRIS consortium (UCLA/IST) for the use of OSIRIS. Computational results were obtained using ARCHIE-WeSt (EPSRC EP/K000586/1). The authors acknowledge funding from U.K. EPSRC (EP/J018171/1, EP/N028694/1), EU H2020 Research and Innovation Programme LASERLAB EUROPE (654148) and EuPRAXIA (653782). Data associated with research published in this paper is available at https://doi.org/10.15129/3e98b6c8-96e6-4c70-b524-6dd5eabea64c.

\section{REFERENCES}

[1] Mittleman, D. M., "Perspective: Terahertz science and technology," Journal of Applied Physics 122, 230901 (Dec. 2017).

[2] Dhillon, S. S., Vitiello, M. S., Linfield, E. H., Davies, A. G., Hoffmann, M. C., Booske, J., Paoloni, C., Gensch, M., Weightman, P., Williams, G. P., Castro-Camus, E., Cumming, D. R. S., Simoens, F., EscorciaCarranza, I., Grant, J., Lucyszyn, S., Kuwata-Gonokami, M., Konishi, K., Koch, M., Schmuttenmaer, C. A., Cocker, T. L., Huber, R., Markelz, A. G., Taylor, Z. D., Wallace, V. P., Axel Zeitler, J., Sibik, J., Korter, T. M., Ellison, B., Rea, S., Goldsmith, P., Cooper, K. B., Appleby, R., Pardo, D., Huggard, P. G., Krozer, V., Shams, H., Fice, M., Renaud, C., Seeds, A., Stöhr, A., Naftaly, M., Ridler, N., Clarke, R., Cunningham, J. E., and Johnston, M. B., "The 2017 terahertz science and technology roadmap," Journal of Physics D: Applied Physics 50, 043001 (Feb. 2017).

[3] Hafez, H. A., Chai, X., Ibrahim, A., Mondal, S., Férachou, D., Ropagnol, X., and Ozaki, T., "Intense terahertz radiation and their applications," Journal of Optics 18, 093004 (Sept. 2016).

[4] Akyildiz, I. F., Jornet, J. M., and Han, C., "Terahertz band: Next frontier for wireless communications," Physical Communication 12, 16-32 (Sept. 2014). 
[5] Zhang, X. C., Shkurinov, A., and Zhang, Y., "Extreme terahertz science," Nature Photonics 11, 16-18 (Jan. 2017).

[6] Wu, Z., Fisher, A. S., Goodfellow, J., Fuchs, M., Daranciang, D., Hogan, M., Loos, H., and Lindenberg, A., "Intense terahertz pulses from SLAC electron beams using coherent transition radiation," Review of Scientific Instruments 84, 022701 (Feb. 2013).

[7] Casalbuoni, S., Schmidt, B., Schmüser, P., and Steffen, B., "Far-Infrared Transition and Diffraction Radiation - Part II: The THz Beamline at the VUV-FEL Linac," Tech. Rep. TESLA-FEL 2006-04 (2006).

[8] Hemsing, E., Stupakov, G., Xiang, D., and Zholents, A., "Beam by design: Laser manipulation of electrons in modern accelerators," Reviews of Modern Physics 86, 897-941 (July 2014).

[9] Esarey, E., Schroeder, C. B., and Leemans, W. P., "Physics of laser-driven plasma-based electron accelerators," Reviews of Modern Physics 81, 1229-1285 (Aug. 2009).

[10] Islam, M. R., Brunetti, E., Shanks, R. P., Ersfeld, B., Issac, R. C., Cipiccia, S., Anania, M. P., Welsh, G. H., Wiggins, S. M., Noble, A., Cairns, R. A., Raj, G., and D A Jaroszynski, "Near-threshold electron injection in the laser-plasma wakefield accelerator leading to femtosecond bunches," New Journal of Physics 17, 093033 (Sept. 2015).

[11] Lundh, O., Lim, J., Rechatin, C., Ammoura, L., Ben-Ismaïl, A., Davoine, X., Gallot, G., Goddet, J.-P., Lefebvre, E., Malka, V., and Faure, J., "Few femtosecond, few kiloampere electron bunch produced by a laser-plasma accelerator," Nature Physics 7, 219-222 (Mar. 2011).

[12] Couperus, J. P., Pausch, R., Köhler, A., Zarini, O., Krämer, J. M., Garten, M., Huebl, A., Gebhardt, R., Helbig, U., Bock, S., Zeil, K., Debus, A., Bussmann, M., Schramm, U., and Irman, A., "Demonstration of a beam loaded nanocoulomb-class laser wakefield accelerator," Nature Communications 8 (Dec. 2017).

[13] Yang, X., Brunetti, E., Gil, D. R., Welsh, G. H., Li, F. Y., Cipiccia, S., Ersfeld, B., Grant, D. W., Grant, P. A., Islam, M. R., Tooley, M. P., Vieux, G., Wiggins, S. M., Sheng, Z. M., and Jaroszynski, D. A., "Three electron beams from a laser-plasma wakefield accelerator and the energy apportioning question," Scientific Reports 7, 43910 (Mar. 2017).

[14] Brunetti, E., Yang, X., Li, F. Y., Reboredo Gil, D., Welsh, G. H., Cipiccia, S., Ersfeld, B., Grant, D. W., Grant, P. A., Islam, M. R., Shahzad, M., Tooley, M. P., Vieux, G., Wiggins, S. M., Sheng, Z. M., and Jaroszynski, D. A., "Wide-angle electron beams from laser-wakefield accelerators," 102400P (May 2017).

[15] Yang, X., Brunetti, E., and Jaroszynski, D. A., "High-energy coherent terahertz radiation emitted by wideangle electron beams from a laser-wakefield accelerator," New Journal of Physics 20, 043046 (Apr. 2018).

[16] Fonseca, R. A., Silva, L. O., Tsung, F. S., Decyk, V. K., Lu, W., Ren, C., Mori, W. B., Deng, S., Lee, S., Katsouleas, T., and Adam, J. C., "OSIRIS: A Three-Dimensional, Fully Relativistic Particle in Cell Code for Modeling Plasma Based Accelerators," in [Computational Science - ICCS 2002], Goos, G., Hartmanis, J., van Leeuwen, J., Sloot, P. M. A., Hoekstra, A. G., Tan, C. J. K., and Dongarra, J. J., eds., 2331, 342-351, Springer Berlin Heidelberg, Berlin, Heidelberg (2002).

[17] Shkvarunets, A. G. and Fiorito, R. B., "Vector electromagnetic theory of transition and diffraction radiation with application to the measurement of longitudinal bunch size," Physical Review Special Topics Accelerators and Beams 11 (Jan. 2008).

[18] Shibata, Y., Ishi, K., Takahashi, T., Kanai, T., Arai, F., Kimura, S.-i., Ohsaka, T., Ikezawa, M., Kondo, Y., Kato, R., Urasawa, S., Nakazato, T., Niwano, S., Yoshioka, M., and Oyamada, M., "Coherent transition radiation in the far-infrared region," Physical Review E 49, 785-793 (Jan. 1994).

[19] Pompili, R., Anania, M. P., Chiadroni, E., Cianchi, A., Ferrario, M., Lollo, V., Notargiacomo, A., Picardi, L., Ronsivalle, C., Rosenzweig, J. B., Shpakov, V., and Vannozzi, A., "Compact and tunable focusing device for plasma wakefield acceleration," Review of Scientific Instruments 89, 033302 (Mar. 2018).

[20] Lindstrøm, C. A., Adli, E., Boyle, G., Corsini, R., Dyson, A. E., Farabolini, W., Hooker, S. M., Meisel, M., Osterhoff, J., Röckemann, J.-H., Schaper, L., and Sjobak, K. N., "Emittance Preservation in an AberrationFree Active Plasma Lens," Physical Review Letters 121 (Nov. 2018).

[21] Pompili, R., Anania, M. P., Bellaveglia, M., Biagioni, A., Bini, S., Bisesto, F., Brentegani, E., Cardelli, F., Castorina, G., Chiadroni, E., Cianchi, A., Coiro, O., Costa, G., Croia, M., Di Giovenale, D., Ferrario, M., Filippi, F., Giribono, A., Lollo, V., Marocchino, A., Marongiu, M., Martinelli, V., Mostacci, A., Pellegrini, D., Piersanti, L., Di Pirro, G., Romeo, S., Rossi, A. R., Scifo, J., Shpakov, V., Stella, A., Vaccarezza, 
C., Villa, F., and Zigler, A., "Focusing of High-Brightness Electron Beams with Active-Plasma Lenses," Physical Review Letters 121 (Oct. 2018).

[22] Leemans, W. P., Geddes, C. G. R., Faure, J., Tóth, C., van Tilborg, J., Schroeder, C. B., Esarey, E., Fubiani, G., Auerbach, D., Marcelis, B., Carnahan, M. A., Kaindl, R. A., Byrd, J., and Martin, M. C., "Observation of Terahertz Emission from a Laser-Plasma Accelerated Electron Bunch Crossing a PlasmaVacuum Boundary," Physical Review Letters 91 (Aug. 2003). 